\title{
Recent Developments in Applications of Quantum-Dot Based Light-Emitting Diodes
}

\author{
Anca Armăşelu \\ Additional information is available at the end of the chapter
}

http://dx.doi.org/10.5772/intechopen.69177

\begin{abstract}
Quantum dot-based light-emitting diodes (QD-LEDs) represent a form of light-emitting technology and are regarded like a next generation of display technology after the organic light-emitting diodes (OLEDs) display. QD-LEDs are different from liquid crystal displays (LCDs), OLEDs, and plasma displays due to the fact that QD-LEDs present an ideal blend of high brightness, efficiency with long lifetime, flexibility, and low-processing cost of organic LEDs. So, QD-LEDs show theoretical performance limits which surpass all other display technologies. The goal of this chapter is, firstly, to provide a historical prospective study of QD-LEDs applications in display and lighting technologies, secondly, to present the most recent improvements in this field, and finally, to discuss about some current directions in QD-LEDs research that concentrate on the realization of the next-generation displays and high-quality lighting with superior color gamut, higher efficiency, and high color rendering index.
\end{abstract}

Keywords: quantum dots, quantum dot-based light-emitting diodes, display technology, lighting technology

\section{Introduction}

Quantum dots (QDs) have attracted interest in the fields of optical applications such as quantum computing, biological, and chemical applications.

In contradistinction to the traditional fluorophores, QDs have unique optical and electronic features, which comprise high quantum yields, high molar extinction coefficients, large effective Stokes shifts [1, 2], broad excitation profiles, narrow/symmetric emission spectra, high resistance to reactive oxygen-mediated photobleaching [2,3], and are against metabolic degradation $[4,5]$. 
QDs are fluorophore nanocrystals whose excitation and emission is basically distinct than classical organic fluorophores [2]. The unique properties of QDs appear almost exclusively due to the size regime in which they exist [6]. QDs obey the quantum mechanical principle of three-dimensional confinement of the charge carriers (electrons, holes) that determine novel quantum phenomena and tunable optical properties, which are sensitive to the size, shape, and material composition of the QDs [7].

QDs have an intrinsic energy bandgap that decides required wavelength of radiation absorption and emission spectra. The bandgap energy increases with the decrease in the dimension of the QD [8]. The color of the light which a QD emits is directly connected to its size; the bigger dots cause longer wavelengths, lower frequencies, and redder light while the smallest dots produce shorter wavelengths, higher frequencies, and bluer light [9-11]. This dimension dependence permits the modulating of the bandgap energy by varying the size of the QD [6, 12].

The wavelength of fluorescence depends on the bandgap, and consequently, it is determined by the dimension of the QD $[6,12]$. The ability to adjust and to control the size of the QDs is relevant and advantageous for many new applications.

Due to their highly tunable properties, QDs have an abundance of applications in a diversity of fields. QD could offer a choice for commercial applications such as display technology. QDs are also brighter than a competing technology that is known as organic light-emitting diode (OLED) displays and could eventually make OLED displays outdated [9]. An OLED is fabricated with organic compounds that light up when fed electricity [13].

An important drawback of the OLED technology is the lack of trust and cheap patterning methods for various color pixels [14]. Because OLEDs are composed of small molecule organics, they are not consistent with the classical lithographical patterning techniques that necessitate exposure to solvents which completely deteriorate the structures of OLED [14]. QDs are the reliable solutions for flat-panel TV screens, digital cameras, mobile phones, and personal gaming equipments because QDs could assist large, flexible displays and would not deteriorate as easily as OLEDs [15].

According to the synthetic method, the QDs can be categorized into epitaxial and colloidal QDs. Colloidal QDs are made through chemical synthesis and are composed of a small inorganic semiconductor core, an inorganic semiconductor shell with a broader bandgap, and a coating of organic passivation ligands $[16,17]$. The optical properties of the original QDs, which contain a core, can be improved by using the coating of higher bandgap materials or passivation of the exterior part of the core [16, 18]. QD technology is used to filter light from light-emitting diodes (LEDs) to backlit liquid crystal displays (LCDs). With the recent enhancements introduced by the usage of the QDs to backlighting technology, LED/LCD TVs are much better today than they were just few years ago [13].

The use of QDs for the improvement of the LED backlighting leads to the enhancement of the useful light throughput and to the providing of a better color gamut [15]. This QD technology system sends the light from a blue LED through the medium of a tube filled with red and green QDs, so that at the other end of the tube, this blend of blue, green, and red light incurs less absorption of unwished colors by the color filters behind the LCD screen $[13,19]$. The 
first TV manufacturer that presented the achievement of this new type of technology called Triluminos quantum dot display technology was Sony in 2013 [13].

A classic light-emitting diode (LED) is made of some materials that are chosen to emit the required color light and are arranged in layers in a structure named a device stack. The dimension of the total width of this device stack is around $10 \mu \mathrm{m}$ [21].

\section{Evolution of quantum dot-based light-emitting diodes}

Due to the multiple advantages of using QDs and their applications in optoelectronic instruments like LEDs, the scientists have created quantum dot-based light-emitting diode (QD-LED) with the improved efficiency and flexibility. QD-LED represents the following generation's display technology after OLED displays [20-22]. QD-LEDs are a form of lightemitting technology for creating large-area displays that could have applications for TVs, cell phones, and digital cameras [15, 20-22].

The structure of QD-LED is analogous to the fundamental design of OLED, with the difference that the light emitting is the QDs, such as cadmium selenide (CdSe) nanocrystals [20, 21].

A classical QD-LED is composed of three layers: one inner layer of QDs as an emissive layer, one outer layer that transports electrons, and one outer layer that transports holes. After applying an electric field on the outside layers, electrons and holes shift in the layer of QD, where they are captured by QD and recombine, emitting photons [22]. Due to the multiple advantages of using the colloidal QDs, the colloidal QDs are a promising way for making QD-LEDs. A great effect of an increased recombination efficiency is obtained by constructing an emissive layer in a single layer of QDs, so that the electrons and holes may be moved directly from the surfaces of electron-transport layer and hole-transport layer [15, 20, 21]. For the definition of the performance of a QD-LED is used the external quantum efficiency (EQE), which is the term that designates the number of photons emitted from the device per electron. For the methodical progress of QD chemistries and active layer designs as well as new device architectures for high-performance QD-LEDs, it is important to discover and to analyze the fundamental causes of inefficiency and to suggest potential solutions [23].

The investigation of the efficiency of the light generation process in the QD-LEDs is an important criterion for achieving high-performance QD-LEDs [24-26].

In 1907, the British scientist named Henry Joseph Round reported light emission from a crystal detector; thus, the idea of light-emitting diode was introduced [27, 28]. In 1927, the Russian researcher Oleg Vladimirovich Losev published a paper about the first light-emitting diode $[27,29]$.

In 1955, Rubin Braunstein, who worked at Radio Corporation of America, discovered that some common diodes emit infrared light when connected to a current [30, 31]. Also, Rubin Braunstein and Egon Loebner reported in 1958 a green LED which was realized from a lead antimonde/germanium alloy [32]. In 1962, the researcher Nick Holonyak Jr., who is called 
as "the father of the light-emitting diode," created the first practical visible-spectrum (red) GaAsP LED at General Electric Company in New York [33]. In 1964, IBM introduced the using of LEDs on circuit boards in an early mainframe computer [31].

Thomas P. Pearsall reported the first high-brightness and high efficiency LED in 1976, for utilization with fiber optics in telecommunications [34]. Akasaki et al. [35] made the first blue LED in 1992 based on GaN with efficiency of 1\%.

Shuji Nakamura realized the first high-brightness blue LED in 1979 at Nichia Corporation laboratory, but it was too expensive for commercial use until 1994 [36, 37]. Then, Shuji Nakamura was awarded the 2006 Millennium Technology Prize for the development of a white LED [38]. The possibility of color displays with blue, red, and green LEDs was advantageously accomplished [39]. Isamu Akasaki, Hiroshi Amano, and Shuji Nakamura have won the Nobel Prize in Physics in 2014 for the invention of the blue LED [40].

An actual impediment for the development of the performance of the LED is an insufficient understanding of the contribution of some extrinsic elements, such as non-radiative recombination at surface defects versus intrinsic processes, such as multicarrier Auger recombination [41].

In recent years, there have been a lot of research to enhance the quality of LEDs, so recently were introduced QD-LEDs that have the attractive features which correlate the excited state dynamics of structurally engineered QDs with their emissive performance inside LED.

In this regard, some important results of these efforts are the latest demonstrations of QD-LEDs with achievement surpassing that of fluorescent OLEDs that were seriously investigated for a minimum of two decades [26, 41, 42].

QD-LEDs not only reduce the consumption of energy but also show high color purity. Studies reported that QD-LEDs exhibit the ability to be more than twice as power efficient than OLEDs at the same color purity [43,44]. QD-LEDs have the advantages of foldability and their wide application for next-generation electronic displays and optical communication technology [45].

QD-LEDs exhibit pure and saturated emission colors with narrow bandwidth. In QD-LEDs, the emission color is powerfully directed by the dimension of the used QD due to the confinement effects [44]. It has been proven that QD-LEDs present a $30-40 \%$ luminance efficiency advantage above OLEDs for the same color point [43, 44].

QD-LEDs offer several promising features, such as size-dependent emission wavelength, narrow emission spectrum, high efficiency, flexibility, and low-processing cost of organic lightemitting device [43]. The luminaire manufactural cost is diminished due to the capability to imprint large-area QD-LEDs on ultra-thin transparent or flexible substrates [44].

Alivisatos and his colleagues realized the first QD-LED in 1994 [46]. This kind of QD-LED is consisted of a common bilayer structure including an indium tin oxide (ITO) anode, plain CdSe QDs, a $p$-paraphenylene vinylene (PPV) layer, and Mg cathode. Another paper from two research groups at MIT reported a single-layer CdSe-QD-LED with the nanocrystals embedded into an organic polymer matrix [47]. These devices exhibited a very small value of EQEs of $0.001-0.01 \%[47,48]$ and $0.0005 \%[47,49]$. 
In superior performance QD-LEDs, the utilized QDs are core-shell type with some structure gradient from core to shell and with various capping ligands or mixed emitting coats $[16,49$, 50]. The researchers found different types of QD-LEDs which are divided into four categories of devices based on their design [16, 49, 50]: type I (QD-LEDs with polymer charge transport coats), type II (QD-LEDs with organic small molecule charge transport coats), type III (QD-LEDs which are composed of inorganic charge transport coats), and type IV (QD-LEDs which contain an inorganic metal oxide semiconductor as the electron transport layer-ETLand an organic semiconductor as the hole transport layer-HTL). In 2002, it was discovered the first type II QD-LED, which was made up of a single monolayer of QDs, was sandwiched between two organic thin films [51].

A drawback to be mentioned to the previously indicated study [51] is the utilization of the organic charge support layers in QD-LEDs that creates an unwanted contribution to the light emission of the LED. This undesired emission perturbs the color purity when a saturated monochromatic emission is wanted. Several authors have shown that in certain cases the perturbing emission could be eliminated [51-53] and in other cases, it could be utilized to build an efficacious white QD-LED [54,55]. The researchers established that the highest efficiency devices with the best construction architecture are QD-LEDs from type IV class. This type of QD-LED is a hybrid tool which comprises an inorganic metal oxide semiconductor such as the ETL and an organic semiconductor such as the HTL.

The usage of the inorganic coat produces substantial advantages for device stability in air $[56,57]$ and maximum current density, whereas the use of the organic coat provides a great tunability and an easy processing [16].

In 2007, Anikeeva et al. [58] reported an efficacious spectrally broad electroluminescent QD-LED with spectral emission tunable across the Comission Internationale de l'Eclairage (CIE) color space. More exactly, the authors describe in this paper LEDs with a broad spectral emission which is produced by electroluminescence from a mixed monolayer of red, green, and blue emitting colloidal QDs in a hybrid organic/inorganic QD-LED. Concurrent electroluminescence of numerous color QDs leads to the evolution of tunable LED colors such as white QD-LEDs [58]. The number of QDs colors which can be utilized in a single device is practically boundless; thus, it is possible to obtain higher color rendering and imitate the solar color temperature with the help of QD-LEDs.

Yang and coworkers $[59,60]$ demonstrated a full range of blue, green, and red quantum dotbased light-emitting devices exhibiting EQEs above 10\%. These devices showed low turn-on voltages and saturated pure colors. It has been reported that the values of the lifetimes for the green and red devices are greater than 90,000 and 300,000 h, respectively.

NanoPhotonica is a company that offers advanced and original nanomaterials and manufacturing methods which assure for electronic displays to show a high resolution, pure, bright colors, and an improved efficiency for an important low cost of production. In 2015, NanoPhotonica exhibited enhanced efficiency for blue and green QD-LEDs [60, 61].

The contribution here reported that by using a promising design strategy for QD synthesis and device fabrication methods, a high value of $21 \%$ EQE for the green QD-LEDs and a value 
of $11.2 \%$ EQE for the blue QD-LEDs were discovered. The value of $21 \%$ EQE in the case of the green QD-LED is the greatest recorded efficiency of any color QD-LED and is the same value as in the case of vacuum-deposited red and green OLEDs utilized in AMOLED display technology which is commercially available [49].

In the following section, the current status of the applications of QD-LEDs and a summary of the issues concerning the limiting of the applicability of QD-LEDs are discussed.

With the aim of achieving a well-designed QD-LED appropriate for general lighting applications, two relevant criteria such as color rendering index (CRI) and the correlated color temperature (CCT) must be discussed in this case. The CCT represents a measure of the light source color appearance described by the vicinity chromaticity coordinates at the blackbody's locus as a one number rather than the two needed to specify a chromaticity $[62,63]$. CRI is defined like the measurement of how the colors look under a light source in comparison with sunlight.

QD-LEDs have already exhibited important advantages in general lighting with higher efficiency and better rendering ability [48, 64, 65]. QD-LEDs have enjoyed a lot of attention as promising devices for next-generation displays. Regarding the usage of QD-LEDs in the area of the display technology, one of the most significant parameters for the characterization of the display devices is color gamut [63]. In this field of applications of QD-LEDs the chromaticity diagrams and color gamut standards in order to measure the purity of the color are utilized.

In this regard, it is noteworthy that the International Commission on Illumination (CIE) created the chromaticity diagram named CIE 1931 color space. QD-LED device represents a competitive backlight resolving for the next-generation LCDs because unlike traditional backlight solutions, the QD backlight provides a larger color gamut with a value more than $115 \%$ National Television System Committee (NTSC) in CIE 1931 color space and with a value more than 140\% NTSC in CIE 1976 [62, 65]. These evaluation criteria listed above for QD-LEDs devices should be considered to create attractive next-generation display applications and superior quality lighting applications with better color gamut, higher efficiency, and high CRI $[62,65]$.

\section{Actual applications of quantum dot-based light-emitting diodes}

QD-LEDs have proved impressive outcomes for medical field, lighting, and display applications.

\subsection{Quantum dot-based light-emitting diodes for phototherapy}

In this part, an important and new application of the use of QD-LEDs in phototherapy is summarized [66]. Phototherapy or light therapy consists of the dermal exposure to light for the treatment of the various medical disorders. Light therapy is used to treat the skin disorders (psoriasis, acne vulgaris, eczema, skin cancer, wound healing), neonatal jaundice, circadian rhythm disorders, and tumors. The researchers [66] have created two medical dressings for phototherapy, 
each of the two medical dressings include an occlusive layer and translucent layer. For one medical dressing, QD-LEDs chips are designed within the occlusive layer and covered with a translucent layer, so as to furnish a characteristic wavelength of light for utilization in phototherapy. The second device, which was discovered by the same authors [66], consists of an occlusive layer and a translucent layer with QDs that are enclosed in a layer or both layers.

\subsection{Quantum dots as electroluminescent light sources}

Solid-state lighting (SSL) represents another field in energy and environmental sustainability, in which QDs can have a remarkable price and a great advantage compared to the actual state of the art. Therefore, QDs are considered valuable materials for displays that are suitable for the incorporation into SSL technologies as downconversion phosphors or electroluminescent phosphors [49]. In last years, much research has been achieved on QD-LEDs as the optimal choice for SSL applications [49, 67]. The greatest assets of QD-LEDs as electroluminescent light sources for SSL technologies are their low cost, high efficiency production compatibility, flexible and versatile form factors, and the capacity to be a light source spread over a large area than a point light source. There are some performance products of this kind which become a commercial reality. For example, two UK companies [64] named Cumbria LED lighting company Marl and Manchester-based Nanoco (Nanoco is the global leader in the evolution and the fabrication of the cadmium-free quantum dots-CFQD) performed the world's first CFQD quantum dot LED lighting product, the Orion QD. QDs that are produced by Nanoco are cadmium-free, absorb light in a broad wavelength series, comprising blue, and emit at a color conditioned by their dimension. In this case, light is only converted to where it is needed.

This type of product finds its application in horticulture because the nanoparticle luminaires produce light comprising blue and red but not green that corresponds to the absorption of the chlorophyll, with none of the light it reflects $[49,68]$. Another high-quality example is represented by a device from Zylight, namely the multiple award-winning F8 100 LED Fresnel which is the next generation of Fresnel light and encloses QDs into its fixture [49, 69]. More exactly, in compliance with Zylight, the F8 includes a certain mix of QDs with traditional phosphor and exhibits a value of the CR) of up to 97 and a quality of light adjusted only by traditional sunlight and incandescent bulbs $[69,70]$. It is expected that with the progress of the research in the field of electroluminescent QD-LEDs, these devices bring substantial improvements for the SSL industry.

The recent studies conducted on the LED base next-generation lighting show remarkable results in the area of lighting for the energy saving for the world warming prevention. In this regard, Song [71] realized a multifunctional LED light source as investigation about the lighting installation using the QD. Due to the fact that artificial light is utilized in different areas such as the increase of the efficiency lighting, the rise of the growth of plant, and the prevention of the disease, LED is a great choice to be extensively used as artificial light in factory plant. Moreover, this LED mentioned before is an energy saving-device and diminishes the emissions of the greenhouse gas. The author presented the achievement [71] of a manufactured LED lamp by the use of QDs as phosphor, in order to be applied like an illumination technology for plant growth used in a multi-wavelength QD-LED device. 
In 2013, Pickett et al. [72] realized an invention that has to do with the QD-LEDs useful for plant, algae, and bacterial growth applications. To overcome the disadvantages of using LEDs, the inventors have suggested the utilization of QD-LEDs as the optimal choice. Thus, the researchers presented in their work that QD-LEDs utilized a primary light source which is a solid-state LED, with blue or UV light, and a secondary light source (which downconverts the primary light) that comprises one or more QD components. In their paper, Pickett et al. [72] described that for the optimization of the plant growth in the agricultural and horticultural field, for the improvement of the process of the growth of algae, and for the stimulation of the photosynthetic bacterial growth in bioremediation goals, the QD-LED lighting systems are utilized. In contrast to the solid-state LED lighting, the QD-LED lighting system offers a less costly choice, emits less heat which could harm the plants and other photosynthetic organisms, can offer a greater light intensity, emits light at wavelengths better geared toward the promotion of the growth of bacteria and algae, thereby minimizing energy losses, and exhibits a high-energy efficiency. Thus, the value of the energy efficiency of QD-LEDs used in this case is in the range of $30-70 \mathrm{~lm} / \mathrm{W}$, in contrast to $10-18 \mathrm{~lm} / \mathrm{W}$ for incandescent bulbs and 35-60 lm/W for fluorescent lamps. Another important advantage of the use of the QD-LED lighting systems is that due to the easy wavelength adjustment of QDs, the emission wavelength of the QD-LED can be simply changed to correspond to a diversity of various photosynthetic bacteria. The QD-LED systems described in this invention can be utilized in a wide variety of applications.

There are two kinds of QD-LEDs, and the dissimilates between these two kinds of QD-LEDs are that the first category is based on photo-excited QDs (photoluminescence QD-LEDs) and the second category of QD-LEDs relies on electro-excited QDs (electroluminescence QD-LEDs) [62]. The most frequently utilized type of QD-LEDs in applications is the photoluminescence QD-LEDs.

Klimov and colleagues [41, 73, 74] from the Nanotechnology and Advanced Spectroscopy Team at Los Alamos National Laboratory reported some substantial advances in the domain of the applications of QD-LEDs. Klimov believes that QD-LEDs can probably offer many benefits over traditional lighting technologies, such as incandescent bulbs, particularly in the fields of efficiency, functioning lifetime, and the color quality of the emitted light. Advanced investigations, made by Klimov and his team from Los Alamos, allow that less wasteful fluorescent light sources quickly substitute the incandescent bulbs, which are known for the conversion of only $10 \%$ of electricity into light and the loss of $90 \%$ of it to heat.

The researchers conducted some spectroscopic studies on the QD-LEDs [41, 73, 74] and have shown that the so-called Auger recombination effect has heavily influenced both LED efficiency and the onset of efficiency roll-off at high currents. The team of researchers established two methods that diminished this issue by using hetero-structured quantum dots.

In the last years, there has been much study in the field of LEDs and photovoltaic solar cells (PV SC). Though OLEDs have the guarantee to overcome the traditional LEDs in performance, OLED materials and manufactural processes of them are not sufficiently advanced to offer this economically [75]. By the evolving a hybrid tool, the efficiency can be increased 
and the manufactural price can potentially be reduced because this hybrid material system is compatible with inexpensive fabrication procedure such as solution processing and rollto-roll deposition and with patterning methods, enabling multicolor light sources to be prepared on the same substrate by replacing the emissive colloidal QD coating [14, 75].

For example, McCreary developed a hybrid device by combining QDs with conjugated polymers to create a QD-LED. The motivation why this design was chosen is to be able to inkjet print the entire tool, at least the polymer and QD layers [75]. The researcher proposed a structure of the hybrid device which is of type ITO/PEDOT/CdSe QD/Au and is shown in Figure 1(A) [75]. In Figure 1(B) the energy bandgap structure for the same tool is presented [75].
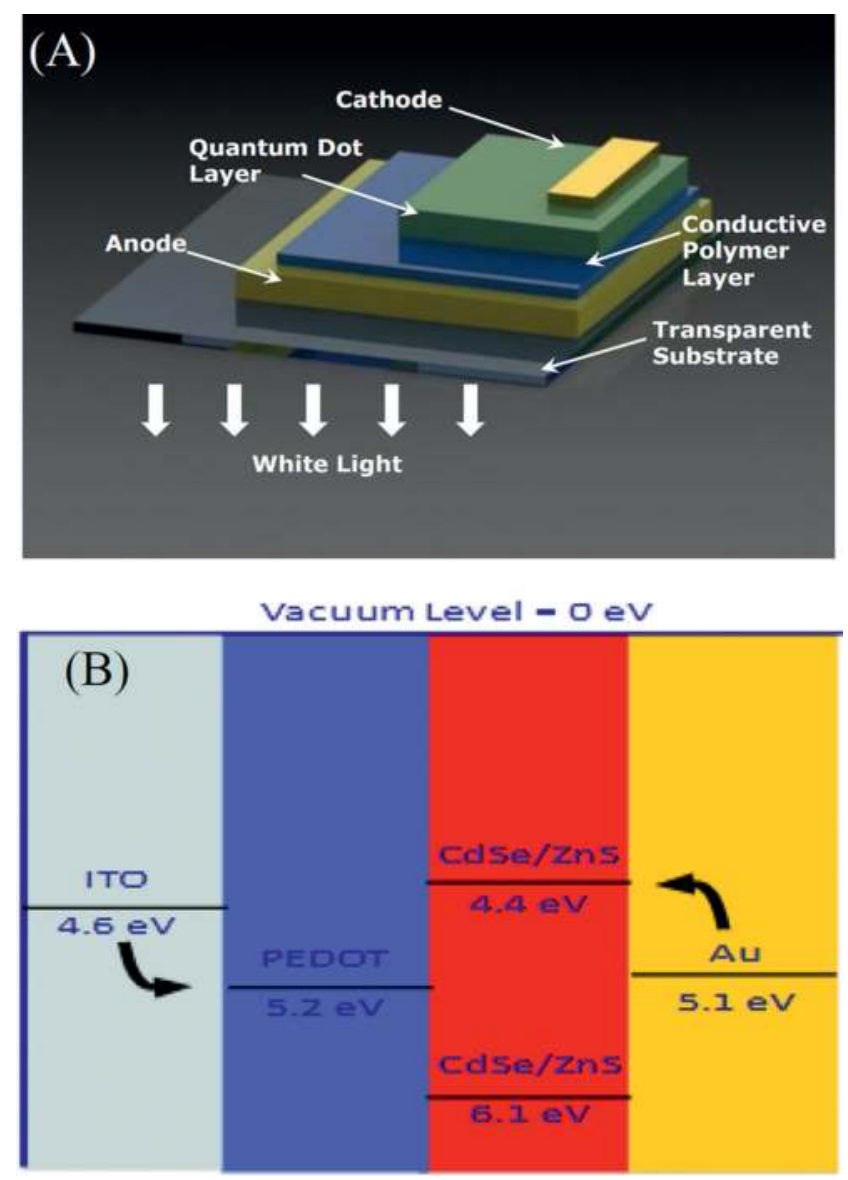

Figure 1. The tool structure of a hybrid LED. (A) Three-dimensional description of the proposed QD-LED [75]. (B) An energy bandgap scheme of the proposed QD-LED and the suggested materials that were used to construct this type of QD-LED [75]. 
Also, the same author explained the manufacture of a hybrid LED with the structure ITO/ PEDOT: PSS/PVK/CdSe QD/Alq3/Al [75]. In order to ease proper hole transport and adequate QD coating, the researcher used a PVK/QD composite solution to make a monolayer layer of QDs using phase separation of the solutes in solution.

This physical modeling of hybrid QD-LEDs of this type, such as those mentioned before [75], makes them applicable to a diversity of hybrid organic QD optoelectronics tools like LEDs, solar cells, photodetectors, and chemical sensors.

\subsection{Quantum dot-based light-emitting diodes for near-field scanning optical microscopy}

QD-LEDs manufactured on silicon have the potential to be used in nanophotonics, optical micro/nanoelectromechanical systems (MEMS/NEMS), and micrototal analysis systems for real-time biomedical screening [21, 76]. QD-LEDs are of considerable interest for new optoelectronic applications such as that which comprise near-field microscopy beyond the diffraction limit, MEMS-based medical endoscopes for sub-cellular imaging, and compact light-on-chip biosensor and biochips [21].

In his paper, Zhu has investigated the case of a new thin film LED device utilizing nanocrystalline silicon QDs as an emission layer and metal oxide as charge transport layers [77]. Silicon (Si) is notably less costly in comparison with materials like germanium or gallium that are applied for commercial SSL devices and is relatively non-toxic as compared to heavy metal like $\mathrm{Cd}$ or $\mathrm{Pb}$. The author developed a thin film LED structure which is based on colloidal silicon nanocrystals using nickel oxide $(\mathrm{NiO})$ and zinc oxide $(\mathrm{ZnO})$ as charge transport layers. The tool that was reported by the researcher is depicted in Figure 2 [77]. ITO represents the anode, and Aluminum ( $\mathrm{Al})$ acts as the cathode. The light is produced when electrons and holes radiatively combine in the silicon nanocrystals (ncSi).

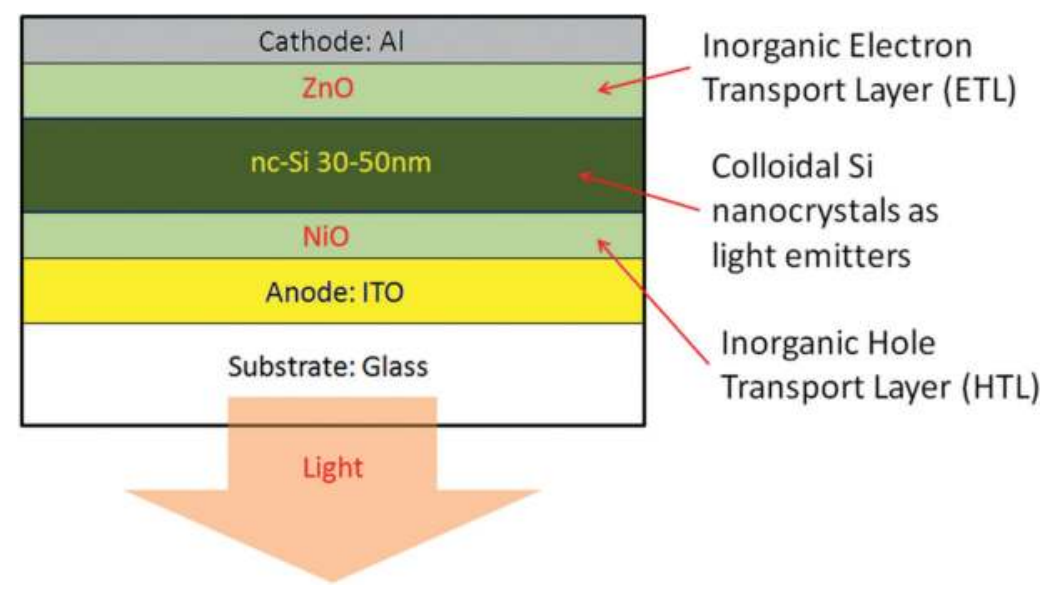

Figure 2. ncSiLED structure with metal oxide charge transport layers [77]. 
Zhu provided an alternative to organic charge transport layers in OLEDs, demonstrating that metal oxide transport layers based on $\mathrm{NiO}$ and $\mathrm{ZnO}$ are electrically more conductive than organic charge transport layers regularly found in OLEDs.

Nanocrystalline silicon QDs exhibited tunable luminescent colors. In this chapter, the author showed that nanocrystalline silicon QDs represent a feasible, truly benign, and ecological substitute for the heavy metal $(\mathrm{Cd}, \mathrm{Pb})$ QDs, without altering in any way the optical properties [77].

There are a lot of studies that demonstrated a number of procedures of creating QD-LEDs through microcontact printing of QDs on a micromachined silicon probe and resulting in this way a novel generation of highly integrated nano-scale optical fluorescent microscopy $[76,78]$. The use of this innovative technology permits to detect the variation of sub-cellular characteristics and to measure the absorption at various wavelengths upon the near-field lighting of individual tumor cells with the aim of the identification of the cancer developmental phase [76].

Another important work in the same area of applications of QD-LEDs relates to the nearfield scanning optical microscopy (NSOM) areas of modern investigation with a QD-LED incorporated at the tip of a scanning probe. Hoshino et al. [79] proved near-field fluorescence excitation and imaging using a QD-LED integrated at the tip of a scanning probe. Also, because QD-LEDs have unique properties like well-controlled emission wavelength and narrow bandwidth, they represent a great choice as excitation sources for fluorescence imaging.

In other works $[80,81]$, the same researchers declared a microcontact printing method which is used to obtain some patterned QD-LEDs on flat silicon substrates. It has been observed that this method is very profitable owing to the connection of the methodology to build siliconbased electronics and MEMS. Hoshino and his team of researchers [79] proved a fluorescence imaging technique, showing that the sensitivity of fluorescence intensity to the QD-LED-QD specimen distance was evaluated down to $50 \mathrm{~nm}$ in order. This procedure might be expanded for the unique molecular order measurements $[79,82]$.

Zhang et al. [83] described a method for the development of a new near-field scanning probe with sub-diffraction-limit resolution by producing a nanometer-sized light source on a patterned probe tip and for the use of the probe in order to detect the molecular signatures of the tumors of the breast. In this project, the authors presented scanning fluorescence imaging with a nano-scale light-emitting diode incorporated at the tip of a silicon microprobe. More accurately, the researchers constructed and studied the QD-LED for bioimaging applications and examined the fluorescence imaging with the on-probe nano-scale QD-LED. It has been shown that QD-LEDs function like near-field excitation sources to enlighten fluorescently labeled cancer cells such as breast cancer cells named MDA435, prostate cancer cells called PC3, and circulating tumor cells in blood. In this chapter [81], a QD-LED at the tip of a micromachined silicon scanning probe was built; the very small size of the QD-LED makes it usable mainly for sub-wavelength optical measurements like in the case of NSOM measurements. 


\section{Conclusions}

The area of QD-LED technology has made immense strides in the past 10 years and the demand for lower price and higher efficiency devices with raised functionality will continue to lead the novelty.

Among all the emerging display and lighting technologies briefly considered in this chapter, QD-LED technologies are by far the most nascent. Like technology, it is a direct challenge to OLEDs.

In this chapter, the recent developments in applications of QD-LEDs and some QD-LEDs qualities in display and lighting applications including their color tunability, durability, and high luminescence efficiency have been reviewed and discussed.

In this work, unique features of QD-LEDs applications for fundamental research and industry which will indubitably spectacularly bring novel design possibilities for the next-generation displays and solid-state lighting in the years to come are presented.

\section{Author details}

Anca Armăşelu

Address all correspondence to: anca_armaselu@yahoo.com

Department of Electrical Engineering and Applied Physics, Faculty of Electrical Engineering and Computer Science, Transilvania University of Brasov, Brasov, Romania

\section{References}

[1] Mazumder S, Dey R, Mitra MK, Mukherjee S, Das GC. Review: Biofunctionalized quantum dots in biology and medicine. Journal of Nanomaterials. 2009;2009:1-17. DOI: $10.1155 / 2009 / 815734$

[2] Deerinck TJ. The application of fluorescent quantum dots to confocal, multiphoton and electron microscopic imaging. Toxicologic Pathology. 2008;36(1):112-116. DOI: 10. $1177 / 0192623307310950$

[3] Medintz IL, Uyeda HT, Goldman ER, Mattoussi H. Quantum dot bioconjugates for imaging, labelling and sensing. Nature Materials. 2005;4:435-446. DOI: 10.1038/nmat1390

[4] Jaiswal JK, Mattoussi H, Mauro JM, Simon SM. Long-term multiple color imaging of live cells using quantum dot bioconjugates. Nature Biotechnology. 2003;21(1):47-51. DOI: $10.1038 /$ nbt767

[5] Ballou B, Lagerholm BC, Ernst LA, Bruchez MP, Waggoner AS. Noninvasive imaging of quantum dots in mice. Bioconjugate Chemistry. 2004;15(1):79-86. DOI: 10.1021/bc03453y 
[6] Melville J. Optical properties of quantum dots [final paper]. UC Berkeley College of Chemistry; 2015. Available from: https://www.ocf.berkeley.edu/ jmlvll/lab-reports/ quantumDots/quantumDots.pdf

[7] Moody G. Confinement effects on the electronic and optical properties of semiconductor quantum dots revelead with two-dimensional coherent spectroscopy [thesis]. University of Colorado; 2013. Available from: https:/jila.colorado.edu/sites/default/files/assets/ files/publications/Thesis_moodyg.pdf

[8] McDaniel J. Quantum Dots: Science and Applications. Available from: http://ion.chem. usu.edu/ tapaskar/James-Quantum\%20Dots\%20Seminar.pdf

[9] Woodford C. What are Quantum Dots? [Internet]. 2016. Available from: http://www. explainthatstuff.com/quantum-dots.html

[10] Parak WJ, Pellegrino T, Plank C. Labelling of cells with quantum dots. Nanotechnology. 2005;16(2):9-25. DOI: 10.1088/0957-4484/16/2/R01

[11] Kippeny T, Swafford LA, Rosenthal SJ. Semiconductor nanocrystals: A powerful visual aid for introducing the particle in a box. Journal of Chemical Education. 2002;79(9):10941100. DOI: 10.1021/ed079p1094

[12] Parak WJ, Manna L, Rimmel Ch, Gerion D, Alivisatos P. Quantum dots. In: Schmid G, editor. Nanoparticles-From Theory to Application. Weinheim: John Wiley and Sons; 2004. 447 p. ISBN: 3-527-30507-6

[13] Denison C. OLED vs LED: Which is the Better TV Technology? [Internet]. 2016. Available from: http://www.digitaltrends.com/home-theater/oled-vs-led-which-is-the better-tvtechnology/

[14] Anikeeva PO. Physical properties and design of light-emitting devices based on organic materials and nanoparticle [thesis]. Massachusetts Institute of Technology; 2009. Available from: https://nanohub.org/resources/9435/download/Polly_thesis.pdf

[15] Quantum Dot Display [Internet]. 2013. Available from: https://en.wikipedia.org/wiki/ Quantum_dot_display\#cite_note-New_Scientist-1

[16] Shirasaki Y, Supran GJ, Bawendi MG, Bulović V. Emergence of colloidal quantum-dot light-emitting technologies. Nature Photonics. 2013;7:13-23. DOI: 10.1038/nphoton. 2012.328

[17] Amini P, Dolatyari M, Rostami G, Rostami A. High througput quantum dot based LEDs. In: Eissa MM, editor. Energy Efficiency Improvements in Smart Grid Components. Rijeka: InTech; 2015. pp. 293-324. DOI: 10.5772/59092

[18] Kim S, Im SH, Kim SW. Performance of light-emitting-diode based on quantum dots. Nanoscale. 2013;5:5205-5214. DOI: 10.1039/C3NR00496A

[19] Perry TS. CES 2015: What the Heck are Quantum Dots? [Internet]. 2015. Available from: http://spectrum.ieee.org/tech-talk/consumer-electronics/audiovideo/what-theheck-are-quantum-dots 
[20] NanoPhotonica [Internet]. 2015. Available from: http://nanophotonica.net/technology/ toggle-id-2

[21] Hussain Z. Introduction to Nanobiotechnology [Internet]. 2015. Available from: http:// www.slideshare.net/zohaibkhan404/quantum-dot-light-emitting-diode

[22] Zyga L. Quantum Dot LED Approaches Theoretical Maximum Efficiency [Internet]. 2013. Available from: https://phys.org/news/2013-05-quantum-dot-approaches-theoretical-maximum.html

[23] Bozyigit D, Wood V. Challenges and solutions for high-efficiency quantum dot-based LEDs. MRS Bulletin. 2013;38:731-736. DOI: 10.1557/mrs.2013.180

[24] Anikeeva PO, Madigan CF, Halpert JE, Bawendi MG, Bulović V. Electronic and excitonic processes in light-emitting devices based on organic materials and colloidal devices based on organic materials and colloidal quantum dots. Physical Review B. 2008;78(8):085434-085441. DOI: 10.1103/PhysRevB.78.085434

[25] Wood V, Panzer MJ, Halpert JE, Caruge J-M, Bawendi MG, Bulović V. Selection of metal oxide charge transport layers for colloidal quantum dot LEDs. ACS Nano. 2009;3(11):3581-3586. DOI:10.1021/nn901074r

[26] Mashford BS, Stevenson M, Popovic Z, Hamilton C, Zhou Z, Breen C, Steckel J, Bulović V, Bawendi M, Coe-Sullivan S, Kazlas PT. High-efficiency quantum-dot light emitting devices with enhance charge injection. Nature Photonics. 2013;7:407-412. DOI: 10.1038/ nphoton.2013.70

[27] Wac Lighting. Responsible Lighting. LED/OLED: Technical Training and Applications [Internet]. 2013. Available from: http://iie.ciapr.org/actividades/seminarios/2009/Presentacion LED-OLED.pdf

[28] Round HJ. A note on carborundum. Electrical World. 1907;19:309-310

[29] Losev OV. Luminous carborundum (silicon carbide) detector and detection with crystals. Telegrafiya i Telefoniya bez Provodov. 1927;44:485-494

[30] Braunstein R. Radiative transitions in semiconductors. Physical Review. 1955;99:1 892-1893

[31] Paisnik K, Rang G, Rang T. Life-time characterization of LEDs. Estonian Journal of Engineering. 2011;17(3):241-251. DOI: 10.3176/eng.2011.3.05

[32] Braunstein R, Loebner EE. Semiconductor device for generating modulated radiation. RCA Corp, assignee, U.S. Patent 3102201 [Issued: 27 August 1963]

[33] Holonyak N Jr, Bevaqua SF. Coherent (visible) light emission from Ga( $\left.\mathrm{As}_{1-\mathrm{x}} \mathrm{P}_{\mathrm{x}}\right)$ junctions. Applied Physics Letters. 1962;1(4):82-83. DOI: 10.1063/1.1753706

[34] Pearsall TP, Miller BI, Capik KJ, Bachmann KJ. Efficient lattice-matched double-heterostructure LED's at 1.1. $\mu \mathrm{m}$ from $\mathrm{Ga}_{x} \mathrm{In}_{1-x} \mathrm{As}_{y} \mathrm{P}_{1-y^{\prime}}$ Applied Physics Letters. 1976;28(9):499501. DOI: $10.1063 / 1.88831$ 
[35] Akasaki I, Amano H, Itoh K, Koide N, Manabe K. GaN-based ultraviolet/blue light emitting devices. Inst. Phys. Conf. Ser. 1992;129:851-856

[36] Nakamura S, Mukai T, Senoh M. Candela-class high-brightness InGaN/AlGaN double-heterostructure blue-light-emitting diodes. Applied Physics Letters. 1994;64: 1687-1689

[37] History of Lighting [Internet]. Available from: http://www.historyoflighting.net/lightbulb-history/history-of-led/

[38] Shuji Nakamura's Millennium Prize a Brilliant Tribute. In: LED Lighting Luminaires and Fixtures. 2006. Available from: http://www.solidstatelightingdesign.com/shujinakamuras-millenium-prize-a-brilliant-tribute/

[39] Muthu S, Schuurmans FPJ, Pashley MD. Red, green, and blue LEDs for white light illumination. IEEE Journal of Quantum Electronics. 2002;8:333-338

[40] Webb J. Invention of Blue LED Wins Physics Nobel [Internet]. 2014. Available from: http://www.bbc.com/news/science-environment-29518521

[41] Bae WK, Park Y-S, Lim J, Lee D, Padilha L, McDaniel H, Robel I, Lee C, Pietryga JM, Klimov VI. Controlling the influence of Auger recombination on the performance of quantum-dot light-emitting diodes. Nature Communications. 2013;4(2661):1-8. DOI: 10.1038/ncomms3661

[42] Kwak J, Bae WK, Lee D, Park I, Lim J, Park M, Cho H, Woo H, Yoon DY, Char K, Lee $\mathrm{S}$, Lee C. Bright and efficient full-color colloidal quantum dot light-emitting diodes using an inverted device structure. Nano Letters. 2012;12(5):2362-2366. DOI: 10.1021/ NL3003254

[43] Altavilla C, Ciliberto E, editors. Inorganic Nanoparticles: Synthesis, Applications, and Perspectives. Boca Raton, FL: Taylor \& Francis; 2011. 547 p. ISBN: $9781439817612-$ CAT\#K10915

[44] Infos and News about QLEDs Quantum Dot Light Emitting Diodes [Internet]. 2013. Available from: http:/www.qled-info.com/introduction/

[45] Yang XY, Mutlugur E, Zhao YB, Gao Y, Leck KS, Ma YY, Ke L, Tan ST, Demir HV, Sun XW. Solution processed tungsten oxide interfacial layer for efficient hole-injection in quantum dot light-emitting diodes. Small. 2014;10(2):247-252. DOI:10.1002/smll.201301199

[46] Colvin VL, Schlamp MC, Alivisatos AP. Light-emitting diodes made from cadmium selenide nanocrystals and a semiconducting polymer. Nature. 1994;370(6488):354-357. DOI: $10.1038 / 370354 \mathrm{a} 0$

[47] Dabboussi BO, Bawendi MG, Onitsuka O, Rubner MF. Electroluminescence from CdSe quantum-dot/polymer composites. Applied Physics Letters. 1995;66(11):1316-1318. DOI: $10.1063 / 1.113227$

[48] Erdem T, Nizamoglu S, Sun XW, Demir HV. A photometric investigation of ultraefficient LEDs with high color rendering index and high luminous efficacy employing 
nanocrystal quantum dot luminophores. Optics Express. 2010;18(1):340-347. DOI: 10.1364/ OE. 18000340

[49] Kitai A, editor. Materials for Solid State Lighting and Displays. Chichester: Wiley; 2017. 384 p. ISBN: 978-1-119-14058-0

[50] Supran GJ. QLEDs for display and solid-state lighting. MRS Bulletin. 2013;3819:703-711. DOI: $10.1557 / \mathrm{mrs} .2013 .181$

[51] Coe S, Woo W-K, Bawendi M, Bulović V. Electroluminescence from single monolayers of nanocrystals in molecular organic devices. Nature. 2002;420(6917):8100-8803. DOI: 10.1038/nature01217

[52] Tan Z, Zhang F, Zhu T, Xu T. Bright and color-saturated emission from blue light-emitting diodes based on solution-processed colloidal nanocrystal quantum dots. Nano Letters. 2007;7(12):3803-3807. DOI: 10.1021/n107230s

[53] Zhu T, Shanmugasundaram K, Price SC, Ruzyllo J, Zhang F, Xu I, Mohney SE, Zhang Q, Wang AY. Mist fabrication of light emitting diodes with colloidal nanocrystal quantum dots. Applied Physics Letters. 2008;92(2):023111-023113. DOI: 10.1063/1.2834734

[54] Li Y, Rizzo A, Mazzeo M, Carbone L, Manna L, Cingolani R, Gigli G. White organic light-emitting devices with CdSe/ZnS quantum dots as a red emitter. Journal of Applied Physics. 2005;97(11):113501. DOI: 10.1063/1.1921341

[55] Chen J, Zhao D, Li C, Xu F, Lei W, Sun L, Nathan A, Sun XW. All solution processed

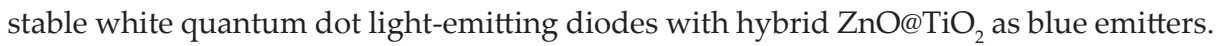
Scientific Reports. 2014;4(4085):1-6. DOI: 10.1038/srep04085

[56] Klein M. Quantum Dots for LED Displays [Internet]. 2014. Available from: http://www. materialsforenergytypepad.com/materials/2014/02/quantum-dots-for-led-displays.html

[57] Kumar B, Campbell SA, Ruden PP. Modeling charge transport in quantum dot light emitting devices with $\mathrm{NiO}$ and $\mathrm{ZnO}$ transport layers and $\mathrm{Si}$ quantum dots. Journal of Applied Physics. 2013;114(044507):1-6. DOI: 10.1063/1.4816680

[58] Anikeeva PO, Halpert JE, Bawendi MG, Bulović V. Electroluminescence from a mixed red-green-blue colloidal quantum dot monolayer. Nano Letters. 2007;7(8):2196-2200

[59] Yang Y, Zheng Y, Cao W, Titov A, Hyvonen J, Manders JR, Xue J, Holloway PH, Qian L. High-efficiency light-emitting devices based on quantum dots with tailored nanostructures. Nature Photonics. 2015;9(4):259-266. DOI: 10.1038/nphoton.2015.36

[60] NanoPhotonica [Internet]. 2015. Available from: http://www.nanophotonica.net/about/

[61] Qin W, Yang Z, Jiang Y, Lam JWY, Liang G, Kwok HS, Tang BZ. Construction of efficient deep blue aggregation-induced emission luminogen from triphenylethene for nondoped organic light-emitting diodes. Chemistry of Materials. 2015;27(11):3892-3901. DOI: 10.1021/acs.chemmater.5b00568 
[62] Xie B, Hu R, Luo X. Quantum dots-converted light-emitting diodes packaging for lighting and display: Status and perspectives. Journal of Electronic Packaging. 2016; 138(2):020803-020803-13. DOI: 10.1115/1.4033143

[63] Lighting Research Center [Internet]. 2004. Available from: http://www.lrc.rpi.edu/programs/nlpip/lightinganswers/lightsources/whatisCCT.asp

[64] Zhong P, He GX, Zhang MH. Optimal spectra of white light-emitting diodes using quantum dot nanophosphors. Optics Express. 2012;20(8):9122-9134. DOI: 10.1364/OE. 20.009122

[65] Luo Z, Chen Y, Wu ST. Wide color gamut LCD with a quantum dot backlight. Optics Express. 2013;21(22):26269-26284. DOI: 10.1364/OE.21.026269

[66] Harris J, Glarvey P, Masala O, Pickett N, Gresty N. Quantum dot light-emitting diodes for phototherapy. Nanoco Technologies, Ltd.; 2014; WO 2014177943 A2. Available from: http://www.patentsencyclopedia.com/app/20140277297

[67] Supran GJS. Enhancing quantum-dot luminescence in visible and infrared light emitting devices [thesis]. Massachusetts Institute of Technology; 2016. Available from: https:// dspace.mit.edu/handle/1721.1/104112

[68] World-First Nano-Particle Luminaires Showcase Growing Benefits [Internet]. 2015. Available from: http//www.en.ofweek.com/new-products/Worl-first-nanoparticleluminaires-showcase-growing-benefits-36576

[69] The Fresnel Light. Reimagined [Internet]. 2017. Available from: http://www.zylight. com/f8-fresnel/

[70] Allard M. Built Like a Tank: The Zylight F8 High Power LED Fresnel Reviewed [Internet]. 2015. Available from: http://www.newsshooter.com/2015/05/01/built -like-a-tank-thezylight-f8-led-fresnel/

[71] Song J-W. Grow light for plant factory using quantum dot LED. Journal of International Council on Electrical Engineering. 2016;6(1):13-16. DOI: 10.1080/22348972.2016.1138604

[72] Pickett N, Nasaani I, Harris J, Gresty N. Quantum dot led's to enhance growth in photosynthetic organism. Nanoco Technologies, Ltd.; 2013; WO 2013150388 A2

[73] Talapin DV, Steckel J. Quantum dot light-emitting devices. MRS Bulletin. 2013;38(9):685691. DOI: 10.1557/mrs.2013.204

[74] Nanoscale Engineering Boosts Performance of Quantum Dot Light Emitting Diodes [Internet]. 2013. Available from: http://esciencenews.com/articles/2013/10/25/nanoscale. engineering.boosts.performance.quantum.dot.light.emitting.diodes

[75] McCreary MZ. Synthesis of cadmium selenide quantum dots for fabrication of hybrid light emitting diodes [thesis]. University of Louisville; 2014. Available from: http:// ir.library.louisville.edu/cgi/viewcontent.cgi?article=1940\&context=etd 
[76] Gopal A, Hoshino K, Zhang JXJ. Quantum dots light emitting devices on MEMS: Microcontact printing, near-field imaging, and early cancer detection. In: Proceedings of SPIE, International Symposium on Photoelectronic Detection and Imaging 2011: Sensor and Micromachined Optical Device Technologies; Vol. 8191; 2011. p. 819106. DOI: 10.1117/12.901089. Available from: http://proceedings.spiedigitallibrary.org/proceeding. aspx?articleid $=1270250$

[77] Zhu J. Nanocrystalline silicon quantum dot light emitting diodes using metal oxide charge transport layers [thesis]. University of Toronto; 2012. Available from: https:// tspace.library.utoronto.ca/bitstream/1807/42432/6/Zhu_Jiayuan_201211_MASc_thesis. pdf

[78] Hoshino K, Gopal A, Zhang X. Contact printing of quantum dot light emitting diode on silicon probe tip. CLEO/QELS: 2010 Laser Science to Photonic Applications; IEEE; 2010. INSPEC Accession Number: 11428025. DOI: 10.1364/CLEO.2010.CTuNN4. Available from: http://ieeexplore.ieee.org/document/5500224/

[79] Hoshino K, Gopal A, Glaz MS, Vanden Bout DA, Zhang X. Nanoscale fluorescence imaging with quantum dot near-field electroluminescence. Applied Physics Letters. 2012;101(4):043118-043118-5. DOI: 10.1063/1.4739235

[80] Gopal A, Hoshino K, Kim S, Zhang X. Multi-color colloidal quantum dot based light emitting diodes micropatterned on silicon. Nanotechnology. 2009;20(23):235201-2352019. DOI: $10.1088 / 0957-4484 / 20 / 23 / 235201$

[81] Gopal A, Hoshino K, Zhan X. Photolithographic patterning of subwavelength top emitting colloidal quantum dot based inorganic light emitting diodes on silicon. Applied Physics Letters. 2010;96(13):131109-131109-3. DOI: 10.1063/1.3373832

[82] Hoshino K, Turner TC, Kim S, Gopal A, Zhang X. Single molecular stamping of sub10-nm colloidal quantum dot array. Langmuir. 2008;24(23):13804-13808. DOI: 10.1021/ la802936h

[83] Zhang XJ, Ferrari M, Cheng M-C. EPDT: Nano-scale light emitting diode on silicon cantilever for near-field microscopy of nanovectors biodistribution in tissues and living cells. Austin, TX, United States: University of Texas. 2011. Available from: http://grantome.com/ghrant/NSF/ECCS-0725886 